\title{
A Bivariate Generalization of Hermite's Interpolation Formula
}

\author{
By A. C. Ahlin
}

\begin{abstract}
One of the most commonly used methods for deriving formulas for bivariate interpolation is that of extending to two variables the formulas of Lagrange, Aitken, Newton, Gauss, Stirling, Everett, Bessel, etc., in which forward, backward and central-differences are used. These formulas have the property that the resulting interpolation polynomial agrees with the interpolated function, $f(x, y)$, at each of the node points of a Cartesian grid. In this study, we shall investigate the existence of a wider class of interpolation formulas, together with their associated error terms, than those obtainable by the method just described. To this end, we develop a bivariate osculatory interpolation polynomial which not only agrees with $f(x, y)$ in function values at each of the node points of a Cartesian grid but which also enjoys the property that agreement in values of partial and mixed partial derivatives up to specified, arbitrary orders is obtainable at these points. The result is essentially a bivariate generalization of Hermite's interpolation formula.
\end{abstract}

1. Introduction. The classical interpolation problem is concerned with approximating a function $f$ at $n$ distinct points, $x_{1}, x_{2}, \cdots, x_{n}$ by a polynomial of degree $n-1$ which has the property that it is in agreement with the function values at each of the points $x$.

In certain cases, values of both $f(x)$ and its derivative $f^{\prime}(x)$ are available at the $n$ points, $x_{1}, \cdots, x_{n}$. In this case, resort can be made to the well-known Hermite interpolation formula (sometimes referred to as the formula for osculating interpolation). Hermite's formula yields a polynomial of degree $2 n-1$ which passes through the given points with given slopes.

The purpose of this paper is to develop an osculatory interpolation polynomial of two variables which agrees with a function, $f(x, y)$, in values of partial ${ }^{*}$ and mixed partial derivatives up to specified, arbitrary orders at each of the $n^{2}$ node points of a rectangular grid formed by the intersection of the vertical lines $x=$ $x_{1}, x_{2}, \cdots, x_{n}$ and the horizontal lines $y=y_{1}, y_{2}, \cdots, y_{n}$.

One would expect that interpolation formulas of the type just described would be considerably more accurate $\dagger$ than ordinary $n^{2}$ point formulas for bivariate interpolation because we have essentially concentrated many more conditions into the same amount of space. Thus, if $N$ conditions are met on the derivatives at each of the $n^{2}$ node points, we may regard our interpolation formula as sort of a confluent form of an $\mathrm{Nn}^{2}$-point formula, having effectively packed the $\mathrm{Nn}^{2}$ argu-

Received June 7, 1963, revised October 22, 1963.

* In this paper whenever we refer to partial derivatives, we shall mean straight partial derivatives as opposed to mixed partial derivatives.

$\dagger$ The truth of this statement becomes evident when one considers, in the univariate case, the relative merits of osculatory interpolation to that of Lagrange interpolation formulas. 
ments into a space containing $n^{2}$ points. This should result in considerably greater accuracy as $n$ increases.

From the point of view of machine computation, an interpolation formula involving only partial derivatives, and not mixed partial derivatives, would appear to be more feasible. This is true because many and varied techniques exist for approximating partial derivatives with respect to $x$ or $y$ over a Cartesian grid. Actual computation merely amounts to holding one variable fixed and using wellknown numerical differentiation formulas to obtain the partial derivatives at each of the grid points.

However, based upon the literature, it appears futile to search for a general bivariate osculatory interpolation formula which contains no mixed partial derivatives and which will fit the function $f(x, y)$ and its first partial derivatives at the node points of a Cartesian grid. For example, Salzer and Kimbro [5] have discovered the surprising fact that for most of the simpler configurations of $\left(x_{i}, y_{i}\right)$, if not in general, it is impossible to fit a polynomial $P(x, y)$ to $f(x, y)$ and its first partial derivatives at a set of arbitrarily chosen points $\left(x_{i}, y_{i}\right)$, even when the total number of conditions invoked at all of the points is exactly equal to the number of coefficients in the general form of the selected $P(x, y)$. Experimenting primarily with complete binary $m-i c$ 's, Salzer and Kimbro found that the only way in which any success at all could be had was to artificially modify the binary $m-i c$ by the addition of one or more higher degree terms, thus relegating the interpolation polynomial to an incomplete binary $m-i c$. Even then, however, they found many exceptional configurations of $\left(x_{i}, y_{i}\right)$ for which no bivariate first order osculatory interpolation (no mixed partial derivative conditions) was possible. For the specialized formulas they have developed, Salzer and Kimbro derived no closed expression remainders but in lieu of this they give formulas for dominant remainder terms.

In this present paper we shall circumvent some of the problems encountered by Salzer and Kimbro by requiring the use of mixed partial derivatives and insisting that all $n^{2}$ node points of the Cartesian grid be utilized. The latter fact is equivalent to saying that our formula will be less general.

Specifically, the purpose of this paper is to generalize the generalization of Hermite's formula in one variable to an analogous formula in two variables $x$ and $y$. We shall include a closed form expression of the error of interpolation.

2. A Uniqueness Theorem. In this section we establish a theorem whose results will be required in subsequent discussion. In proving this theorem we shall rely heavily upon the following lemma due to Walsh [8].

Lемма 1. Let the distinct points $z_{1}, z_{2}, \cdots, z_{k}$ and values $w_{j}{ }^{(0)}, w_{j}{ }^{(1)}, \cdots, w_{j}{ }^{\left(m_{j}\right)}$, $j=1,2, \cdots, k$ be given. Then there exists a unique polynomial $p(z)$ of degree

$$
n=-1+\sum_{j=1}^{k}\left(m_{j}+1\right)
$$

which satisfies the conditions

$$
p^{(v)}\left(z_{j}\right)=w_{j}{ }^{(v)}, \quad v=0,1, \cdots, m_{j} ; j=1,2, \cdots, k .
$$

The theorem which we are about to prove is essentially a two-dimensional analog of the lemma just stated. 
Theorem 1. Let $f_{r, i}^{(p, q)}$ be given numbers where $p$ and $q$ run from 0 to $k-1$ and $r$ and $i$ run from 1 to $n$; let $\left(x_{r}, y_{i}\right)$ be $n^{2}$ distinct points for $1 \leqq r \leqq n, 1 \leqq i \leqq n$. Then there exists a unique polynomial $Q(x, y)$ of degree not exceeding $n k-1$ in $x$ and of degree not exceeding $n k-1$ in $y$ such that

$$
\frac{\partial^{p+q}}{\partial x^{p} \partial y^{q}} Q\left(x_{r}, y_{i}\right)=f_{r, i}^{(p, q)} \quad(p, q=0, \cdots, k-1 ; r, i=1, \cdots, n)
$$

Proof. The system of Equations 1 is a system of $k^{2} n^{2}$ equations in the $k^{2} n^{2}$ unknowns which are the coefficients of $Q(x, y)$. Hence the existence of a solution for these coefficients will be guaranteed if their uniqueness is established, i.e., if the matrix of (1) possesses an inverse.

Suppose that another polynomial, $S(x, y)$, of degree not exceeding $n k-1$ in $x$ and of degree not exceeding $n k-1$ in $y$ and having the desired properties given by (1) does exist. Then $S(x, y)-Q(x, y) \equiv T(x, y)$ is a polynomial of maximum degree $n k-1$ in $x$ and $n k-1$ in $y$ which satisfies the conditions

$$
\frac{\partial^{p+q}}{\partial x^{p} \partial y^{q}} T\left(x_{r}, y_{i}\right)=0
$$

where, as before, $p, q, r$ and $i$ are allowed to take on all values given in (1). For brevity let us make the following definition

$$
U_{p}(x, y) \equiv \frac{\partial^{p}}{\partial x^{p}} T(x, y), \quad(p=0,1, \cdots, k-1) .
$$

If use is made of (1), one notices that along the fixed vertical line $x=x_{r}$,

$$
\frac{\partial^{q}}{\partial y^{q}} U_{p}\left(x_{r}, y_{i}\right)=\frac{\partial^{p+q}}{\partial y^{q} \partial x^{p}} T\left(x_{r}, y_{i}\right)=0 \quad(q=0,1, \cdots, k-1 ; i=1,2, \cdots, n) .
$$

Thus, according to the results of Lemma 1 we deduce directly from (3) the result

$$
\left.\frac{\partial^{p}}{\partial x^{p}} T\left(x_{r}, y\right)=0 \quad \text { (for all } y ; r=1,2, \cdots, n\right) .
$$

We recall that the degree of $T(x, y)$ in $x$ does not exceed $n k-1$. This fact, coupled with the fact that each of the $k$ derivatives in $x$ of $T(x, y)$ vanishes for each of the values $x_{1}, x_{2}, \cdots, x_{n}$, for each value of $y$ (in accordance with (4)) enables us to once again directly apply Lemma 1 to obtain the result

$$
T(x, y) \equiv 0
$$

(for all $x$ and all $y$ )

Thus, $S(x, y)=Q(x, y)$ everywhere.

3. Interpolation for Functions of Two Variables. Patterning an analysis after that due to Walsh [8] we shall develop a polynomial interpolation formula for a function of two complex variables. Let us first make the following definitions:

$$
\hat{\alpha}(z)=\alpha(z)\left(z-z_{n+1}\right)\left(z-z_{n+2}\right) \cdots\left(z-z_{k n}\right)
$$

and

$$
\hat{\beta}(w)=\beta(w)\left(w-w_{n+1}\right)\left(w-w_{n+2}\right) \cdots\left(w-w_{k n}\right)
$$


where

$$
\alpha(z)=\prod_{i=1}^{n}\left(z-z_{i}\right)
$$

and

$$
\beta(w)=\prod_{j=1}^{n}\left(w-w_{j}\right)
$$

Define now a polynomial of degree $n k-1$ in $z$ and $n k-1$ in $w$ as follows

$$
Q(z, w)=\left[\frac{\hat{\alpha}(s)-\hat{\alpha}(z)}{\hat{\alpha}(s)(s-z)}\right]\left[\frac{\hat{\beta}(t)-\hat{\beta}(w)}{\hat{\beta}(t)(t-w)}\right]
$$

Now if the polynomial $Q(z, w)$ (whose coefficients are clearly functions of $s$ and $t$ ) is multiplied by $\left[1 /(2 \pi i)^{2}\right] f(s, t) d s d t$ and integrated with respect to $s$ and $t$ over arbitrary closed Jordan curves $C_{1}$ in the complex $s$-plane and $C_{2}$ in the complex $t$-plane, where $f(z, w)$ is an arbitrary function analytic simultaneously in $z$ and $w$ for $z$ on and within $C_{1}$ and $w$ on and within $C_{2}$, the result is still a polynomial in $z$ and $w$ of degree not exceeding $n k-1$ in either variable. If we choose $C_{1}$ to contain the points $z_{1}, z_{2}, \cdots, z_{k n}$ in its interior and similarly choose $C_{2}$ to contain the points $w_{1}, w_{2}, \cdots, w_{k n}$ in its interior, while $z$ and $w$ lie within or on $C_{1}$ and $C_{2}$ respectively, we obtain the polynomial

$$
\hat{q}(z, w)=\left[\frac{1}{2 \pi i}\right]^{2} \int_{C_{1}} \int_{C_{2}}\left[\frac{\hat{\alpha}(s)-\hat{\alpha}(z)}{\hat{\alpha}(s)(s-z)}\right]\left[\frac{\hat{\beta}(t)-\hat{\beta}(w)}{\hat{\beta}(t)(t-w)}\right] f(s, t) d s d t .
$$

We notice that even if $z$ lies on $C_{1}$ or if $w$ lies on $C_{2}$, the integrand has no singularity for in the limit as $s$ approaches $z$ and/or $t$ approaches $w$, the integrand involves either or both of the terms

$$
\lim _{s \rightarrow z} \frac{\hat{\alpha}(s)-\hat{\alpha}(z)}{\alpha(s)(s-z)}=\frac{\hat{\alpha}^{\prime}(z)}{\hat{\alpha}(z)}
$$

and

$$
\lim _{t \rightarrow w} \frac{\hat{\beta}(t)-\hat{\beta}(w)}{\hat{\beta}(t)(t-w)}=\frac{\hat{\beta}^{\prime}(w)}{\hat{\beta}(w)}
$$

which are finite since $\hat{\alpha}(z) \neq 0$ for $z$ on $C_{1}$ and $\hat{\beta}(w) \neq 0$ for $w$ on $C_{2}$.

Let $C_{1}$ be a closed Jordan curve enclosing a simply connected region of the complex variable $s$ which contains the point $z$. Let $C_{2}$ be a closed Jordan curve enclosing a simply connected region of the complex variable $t$ which contains the point $w$. Then Cauchy's integral formula can be easily extended to give the result

$$
f(z, w)=\left(\frac{1}{2 \pi i}\right)^{2} \int_{c_{1}} \int_{c_{2}} \frac{f(s, t)}{(s-z)(t-w)} d s d t .
$$

With the use of (14) the integrand in (11) may be expanded to obtain the result

$$
\begin{aligned}
f(z, w)-\hat{q}(z, w)=\frac{\hat{\alpha}(z)}{2 \pi i} \int_{C_{1}} \frac{f(s, w)}{\hat{\alpha}(s)(s-z)} d s+\frac{\hat{\beta}(w)}{2 \pi i} \int_{C_{2}} \frac{f(z, t)}{\hat{\beta}(t)(t-w)} d t \\
\\
\quad-\frac{\hat{\alpha}(z) \hat{\beta}(w)}{(2 \pi i)^{2}} \int_{C_{1}} \int_{C_{2}} \frac{f(s, t)}{\hat{\alpha}(s) \hat{\beta}(t)(s-z)(t-w)} d s d t .
\end{aligned}
$$


The right-hand expression of (15) is therefore the error resulting from approximating the function $f(z, w)$ by the polynomial $\hat{q}(z, w)$.

Particular notice of the terms of the right-hand expression of (15) reveal the important fact that $f(z, w)$ and $\hat{q}(z, w)$ coincide at the $k^{2} n^{2}$ point pairs $\left(z_{i}, w_{j}\right)$, ( $1 \leqq i, j \leqq k n)$, whether or not the $z_{i}$ 's and $w_{j}$ 's are distinct for the three contour integrals are all analytic as long as $z$ is interior to $C_{1}$ and $w$ is interior to $C_{2}$, hence analytic at each of the point pairs $\left(z_{i}, w_{j}\right)$ irrespective of the multiplicities of either the $z_{i}$ 's or the $w_{j}$ 's. Consequently, we may consider the limiting case when the points $z_{i}$ and $w_{j}$ nearly coincide with the adoption of the notation

$$
\left.\begin{array}{rl}
z_{N+r} & =z_{r}+\epsilon_{r} \\
w_{N+s} & =w_{s}+\delta_{s}
\end{array}\right\} \quad(N=n, 2 n, \cdots, k n-n ; r, s=1,2, \cdots, n)
$$

Introducing the notation of (16) into (15) with reference to (6) and (7), and letting $\epsilon_{i} \rightarrow 0$ and $\delta_{j} \rightarrow 0$, there follows

$$
\begin{aligned}
f(z, w)-\hat{q}(z, w)= & \frac{[\alpha(z)]^{k}}{2 \pi i} \int_{C_{1}} \frac{f(s, w)}{[\alpha(s)]^{k}(s-z)} d s \\
& +\frac{[\beta(w)]^{k}}{2 \pi i} \int_{C_{2}} \frac{f(z, t)}{[\beta(t)]^{k}(t-w)} d t \\
& \quad-\frac{[\alpha(z) \beta(w)]^{k}}{[2 \pi i]^{2}} \int_{C_{1}} \int_{C_{2}} \frac{f(s, t)}{[\alpha(s) \beta(t)]^{k}(s-z)(t-w)} d s d t .
\end{aligned}
$$

If we specialize (17) to the case where $z, w, z_{i}$ and $w_{j}$ are real by setting $z=x$, $w=y, z_{i}=x_{i}$ and $w_{j}=y_{j},{ }^{*}$ there follows directly

$$
\begin{aligned}
f(x, y)-q(x, y)= & \frac{[\lambda(x)]^{k}}{2 \pi i} \int_{C_{1}} \frac{f(s, y)}{[\lambda(s)]^{k}(s-x)} d s \\
& +\frac{[\mu(y)]^{k}}{2 \pi i} \int_{C_{2}} \frac{f(x, t)}{[\mu(t)]^{k}(t-y)} d t \\
& \quad-\frac{[\lambda(x) \mu(y)]^{k}}{[2 \pi i]^{2}} \int_{C_{1}} \int_{C_{2}} \frac{f(s, t)}{[\lambda(s) \mu(t)]^{k}(s-x)(t-y)} d s d t
\end{aligned}
$$

where we have made use of the defining equations

$$
\lambda(x)=\prod_{i=1}^{n}\left(x-x_{i}\right)
$$

and

$$
\mu(y)=\prod_{j=1}^{n}\left(y-y_{j}\right)
$$

With the specialization of (17) to (18) we should not overlook the rather obvious fact that the values $x_{1}, x_{2}, \cdots, x_{n}$ and $x$ lie interior to $C_{1}$ and the values $y_{1}, y_{2}, \cdots, y_{n}$ and $y$ lie interior to $C_{2}$.

\footnotetext{
* We shall define the polynomial $q(x, y)$ to be that polynomial obtained from $\hat{q}(z, w)$ by the replacement of $z, w, z_{i}$ and $w_{j}$ with the indicated substitutions.
} 
4. The Generalization of Hermite's Formula. We state here the general result which is to be proved.

THEOREm 2. Let $f_{r, i}^{(u, v)} x_{r}$ and $y_{i}$ be given real numbers where $u$ and $v$ run from 0 to $k-1$ and $r$ and $i$ run from 1 to $n$. Then the polynomial $q(x, y)$ of degree not exceeding $n k-1$ in $x$ and of degree not exceeding $n k-1$ in $y$ such that

$$
\left.\frac{\partial^{u+v} q(x, y)}{\partial x^{u} \partial y^{v}}\right|_{x=x_{r}, y=y_{i}}=f_{r, i}^{(u, v)}
$$

is given by

$$
q(x, y)=\frac{[\lambda(x) \mu(y)]^{k}}{[(k-1) !]^{2}} \sum_{i=1}^{n} \sum_{r=1}^{n} \sum_{v=0}^{k-1} \sum_{u=0}^{k-1} M_{i, r, v, u}(x, y) f_{r, i}^{(u, v)}
$$

where

$$
M_{i, r, v, u}(x, y)=\left(\begin{array}{c}
k-1 \\
v
\end{array}\right)\left(\begin{array}{c}
k-1 \\
u
\end{array}\right)\left[D_{s}^{k-1-u} \frac{1}{A_{r}(s)}\right]_{s=x_{r}}\left[D_{t}^{k-1-v} \frac{1}{B_{i}(t)}\right]_{t=y_{i}}
$$

with

$$
\begin{aligned}
A_{r}(s) & =(s-x)\left[\left(s-x_{1}\right)\left(s-x_{2}\right) \cdots\left(s-x_{r-1}\right)\left(s-x_{r+1}\right) \cdots\left(s-x_{n}\right)\right]^{k}, \\
B_{i}(t) & =(t-y)\left[\left(t-y_{1}\right)\left(t-y_{2}\right) \cdots\left(t-y_{i-1}\right)\left(t-y_{i+1}\right) \cdots\left(t-y_{n}\right)\right]^{k}
\end{aligned}
$$

and $D_{s}$ and $D_{t}$ are the operators of differentiation with respect to $s$ and $t$.

Remark. If $q(x, y)$ is the polynomial approximation (interpolation polynomial) to a function $f(x, y)$ then the formula becomes

$$
q(x, y)=\frac{[\lambda(x) \mu(y)]^{k}}{[(k-1) !]^{2}} \sum_{i=1}^{n} \sum_{i=1}^{n} \sum_{v=0}^{k-1} \sum_{u=0}^{k-1} M_{i, r, v, u}(x, y) \frac{\partial^{u+v}}{\partial x^{u} \partial y^{v}} f\left(x_{r}, y_{i}\right)
$$

5. Proof of the Theorem. In proving the theorem we shall rely upon the results already established in Sections 2 and 3. In particular we shall show that application of residue theory from complex variables, when applied to (18), yields the results of the theorem. Finally, Theorem 1 is used to establish the uniqueness of the formula thus found thereby completing the proof.

From elementary theory of complex variables we recall the residue theorem which states that if $g(z)$ is analytic on and within a closed contour $C$, with the exception of a finite number of singular points $z_{1}, z_{2}, \cdots, z_{n}$ interior to $C$, then

$$
\int_{C} g(z) d z=2 \pi i\left(K_{1}+K_{2}+\cdots+K_{n}\right),
$$

where the integral is taken counterclockwise around $C$ where $K_{1}, K_{2}, \cdots, K_{n}$ denote the residues of $g(z)$ at those points. A well-known technique for computing the residues for poles of any order may be found in [2], for example.

Let $f(z, w)$ be a function jointly analytic in $z$ and $w$ for $z$ on and within a contour $C_{1}$ in the complex $s$-plane and for $w$ on and within a contour $C_{2}$ in the complex $t$-plane such that $x_{1}, x_{2}, \cdots, x_{n}$ lie within $C_{1}$ and $y_{1}, y_{2}, \cdots, y_{n}$ lie within $C_{2}$ so that

$$
\left.\frac{\partial^{u+v} f(z, w)}{\partial z^{u} \partial w^{v}}\right|_{z=x_{r}, w=y_{i}}=f_{r, i}^{(u, v)}
$$


For example, $f(z, w)$ could be taken as the polynomial $q(z, w)$ itself, since the existence of $q(z, w)$ has been established in Theorem 1 .

If the integral in the last term of the right-hand expression of (18) is written as

$$
\int_{C_{1}} \frac{1}{[\lambda(s)]^{k}(s-x)}\left[\int_{C_{2}} \frac{f(s, t)}{[\mu(t)]^{k}(t-y)} d t\right] d s,
$$

then the residue theorem may be invoked first upon the integral in the brackets and secondly upon the integral over the closed curve $C_{1}$ in the $s$-plane. Evaluating the first two terms in the right-hand expression of (18) in the obvious manner with the use of the residue theorem and the last term as just indicated, $f(x, y)$ conveniently drops out and one obtains after considerable simplification the result

$$
q(x, y)=\frac{[\lambda(x) \mu(y)]^{k}}{[(k-1) !]^{2}}\left[\sum_{i=1}^{n} \sum_{r=1}^{n} H_{r}^{(i)}\right]
$$

where

$$
H_{r}^{(i)}=\lim _{s \rightarrow x_{r}} \frac{d^{k-1}}{d s^{k-1}}\left\{\frac{\left(s-x_{r}\right)^{k} K_{i}(s, y)}{[\lambda(s)]^{k}(s-x)}\right\}
$$

and

$$
K_{i}(s, y)=\lim _{t \rightarrow y_{i}} \frac{d^{k-1}}{d t^{k-1}}\left\{\frac{\left(t-y_{i}\right)^{k} f(s, t)}{[\mu(t)]^{k}(t-y)}\right\}
$$

Let us adopt the notations

$$
(s-x)[\lambda(s)]^{k} /\left(s-x_{r}\right)^{k}=A_{r}(s)
$$

and

$$
(t-y)[\mu(t)]^{k} /\left(t-y_{i}\right)^{k}=B_{i}(t), \quad(t-y)[\mu(t)]^{k}=\bar{B}_{i}(t)
$$

Thus, since $f(s, t)$ is analytic at $t=y_{i}$ for all $s$ and $\bar{B}_{i}{ }^{(j)}\left(y_{i}\right)=0$ for $j=0,1,2$, $\cdots, k-1, K_{i}(s, y)$ can be written as

$$
\begin{aligned}
& K_{i}(s, y)=\lim _{t \rightarrow y_{i}} \frac{d^{k-1}}{d t^{k-1}} \\
& \cdot\left\{\frac{f\left(s, y_{i}\right)+f^{\prime}\left(s, y_{i}\right)\left(t-y_{i}\right)+\cdots+f^{(n)}\left(s, y_{i}\right)\left(t-y_{i}\right)^{n} / n !+\cdots}{\frac{\bar{B}^{(k)}\left(y_{i}\right)}{k !}+\frac{\bar{B}^{(k+1)}\left(y_{i}\right)\left(t-y_{i}\right)}{(k+1) !}+\cdots+\frac{\bar{B}^{(n k+1)}\left(y_{i}\right)\left(t-y_{i}\right)^{n k+1-k}}{(n k+1) !}}\right\} .
\end{aligned}
$$

Using Leibnitz' formula [4, p. 66] to evaluate the right-hand expression of (23), (or (26)), we obtain

$$
K_{i}(s, y)=\sum_{v=0}^{k-1}\left(\begin{array}{c}
k-1 \\
v
\end{array}\right) \frac{d^{k-1-v}}{d t^{k-1-v}}\left\{\frac{1}{B_{i}\left(y_{i}\right)}\right\} \frac{\partial^{v}}{\partial y^{v}} f\left(s, y_{i}\right)
$$

If we now insert $K_{i}(s, y)$, as given by (27) into the right-hand expression of (22) and recall that $f\left(s, y_{i}\right)$ is analytic at $s=x_{r}$, then Taylor's series and Leibnitz' formula can be used as before to obtain the final expression for $H_{r}{ }^{(i)}$,

$$
\begin{aligned}
H_{r}^{(i)}=\sum_{v=0}^{k-1} \sum_{u=0}^{k-1}\left(\begin{array}{c}
k-1 \\
v
\end{array}\right)\left(\begin{array}{c}
k-1 \\
u
\end{array}\right) D_{t}{ }^{k-1-v}\left\{\frac{1}{B_{i}\left(y_{i}\right)}\right\} \\
\cdot D_{s}{ }^{k-1-u}\left\{\frac{1}{A_{r}\left(x_{r}\right)}\right\} \frac{\partial^{u+v}}{\partial x^{u} \partial y^{v}} f\left(x_{r}, y_{i}\right) \quad(i, r,=1,2, \cdots, n),
\end{aligned}
$$


where $D_{t}$ and $D_{s}$ are derivative operators representing differentiation of $B_{i}(t)^{-1}$ and $A_{r}(s)^{-1}$ with respect to $t$ and $s$ respectively.

Referring now to (18) we notice that $q(x, y)$ is a polynomial of degree $k n-1$ in $x$ and $k n-1$ in $y$ satisfying the $k^{2} n^{2}$ conditions

$$
\left.\frac{\partial^{u+v} q(x, y)}{\partial x^{u} \partial y^{v}}\right|_{x=x_{r}, y=y_{i}}=f_{r, i}^{(u, v)} \quad(u, v=0,1, \cdots, k-1 ; r, i=1,2, \cdots, n) .
$$

Thus in accordance with the results of Theorem 1, we are able to establish the fact that $q(x, y)$ given by $(21)$ and $(28)$ is unique. Moreover, simple inspection of (21) and (28) reveals that the $q(x, y)$ so given is identical in form to that given by the theorem and our proof is complete.

6. Hermite's Formula for Two Variables. We shall now proceed to illustrate the results of the previous section by applying them to the specific case of Hermite's formula for functions of two variables [1, p. 32].

According to (21) and (28), Hermite's formula for functions of two variables is given as

$$
\begin{aligned}
q(x, y)= & {[\lambda(x) \mu(y)]^{2} \sum_{i=1}^{n} \sum_{r=1}^{n}\left[D_{s}\left\{\frac{1}{A_{r}\left(x_{r}\right)}\right\} D_{t}\left\{\frac{1}{B_{i}\left(y_{i}\right)}\right\} f\left(x_{r}, y_{i}\right)\right.} \\
& +\frac{1}{B_{i}\left(y_{i}\right)} D_{s}\left\{\frac{1}{A_{r}\left(x_{r}\right)}\right\} \frac{\partial}{\partial y} f\left(x_{r}, y_{i}\right)+\frac{1}{A_{r}\left(x_{r}\right)} D_{t}\left\{\frac{1}{B_{i}\left(y_{i}\right)}\right\} \frac{\partial}{\partial x} f\left(x_{r}, y_{i}\right) \\
& \left.+\frac{1}{A_{r}\left(x_{r}\right)} \frac{1}{B_{i}\left(y_{i}\right)} \frac{\partial^{2}}{\partial x \partial y} f\left(x_{r}, y_{i}\right)\right]
\end{aligned}
$$

Evaluating the terms involving derivatives by the repeated use of L'Hospitals rule, we obtain, finally

$$
\begin{aligned}
f(x, y) \approx q(x, y)= & \sum_{i=1}^{n} \sum_{r=1}^{n} h_{r}(x) g_{i}(y) f\left(x_{r}, y_{i}\right)+\sum_{i=1}^{n} \sum_{r=1}^{n} h_{r}(x) \bar{g}_{i}(y) \frac{\partial}{\partial y} f\left(x_{r}, y_{i}\right) \\
& +\sum_{i=1}^{n} \sum_{r=1}^{n} \bar{h}_{r}(x) g_{i}(y) \frac{\partial}{\partial x} f\left(x_{r}, y_{i}\right)+\sum_{i=1}^{n} \sum_{r=1}^{n} \bar{h}_{r}(x) \bar{g}_{i}(y) \frac{\partial^{2}}{\partial x \partial y} f\left(x_{r}, y_{i}\right)
\end{aligned}
$$

where we have abbreviated

$$
\begin{aligned}
& h_{r}(x)=\left[1-2 l_{r}{ }^{\prime}\left(x_{r}\right)\left(x-x_{r}\right)\right]\left[1_{r}(x)\right]^{2} \\
& \bar{h}_{r}(x)=\left(x-x_{r}\right)\left[l_{r}(x)\right]^{2} \\
& g_{i}(y)=\left[1-2 m_{i}{ }^{\prime}\left(y_{i}\right)\left(y-y_{i}\right)\right]\left[m_{i}(y)\right]^{2} \\
& \bar{g}_{i}(y)=\left(y-y_{i}\right)\left[m_{i}(y)\right]^{2}
\end{aligned}
$$

and $l_{r}(x)$ and $m_{i}(y)$ are the Lagrangian coefficient functions defined as

$$
\begin{aligned}
l_{r}(x) & =\frac{\lambda(x)}{\left(x-x_{r}\right) \lambda^{\prime}\left(x_{r}\right)} \\
m_{i}(y) & =\frac{\mu(y)}{\left(y-y_{i}\right) \mu^{\prime}\left(y_{i}\right)},
\end{aligned}
$$

where $\lambda(x)$ and $\mu(y)$ are given by (19) and (20). 
It is a simple matter to verify that the four conditions invoked upon $q(x, y)$ in (29) for $u, v=0,1$ at each of the points $x_{r}, y_{i}$ are satisfied by the expression derived for $q(x, y)$ above. Thus, the results of Theorem 1 enable us to say that no other polynomial in $x$ and $y$ of degree $2 n-1$ in each $x$ and $y$ and satisfying the desired conditions, exists.

7. Specialization to Functions of One Variable. Suppose that one wishes to generalize Hermite's formula for functions of one variable such that a polynomial is formed which has the properties that it is in agreement with $f(x), f^{\prime}(x)$, $\cdots, f^{(k-1)}(x)$ at each of the $n$ points $x_{1}, x_{2}, \cdots, x_{n}$. From Lemma 1 we know that only one such polynomial of degree $n k-1$ in $x$ exists.

Using an approach involving the use of contour integrals, and identical in technique to that used in the derivation of Theorem 2 , we obtain the one variable analogue, $p(x)$, of $q(x, y)$

$$
f(x) \approx p(x)=\sum_{i=1}^{n} \sum_{j=0}^{k-1} C_{i j}(x) f^{(j)}\left(x_{i}\right)
$$

where

$$
C_{i j}=-\frac{[\lambda(x)]^{k}}{(k-1) !}\left(\begin{array}{c}
k-1 \\
j
\end{array}\right) D_{s}^{k-1-j}\left\{\frac{1}{\left(A_{i}\left(x_{i}\right)\right.}\right\}
$$

and all the symbols utilized in (30) and (31) have been previously defined. One can easily verify that $p(x)$ in (30) resolves itself into Hermite's formula [4, p. 316] with $k=2$.

Spitzbart [7] has found that $C_{i j}$ in (30) is given by the formula

$$
\hat{C}_{i j}=\frac{[\lambda(x)]^{k}\left(x-x_{i}\right)^{j-k}}{j !} \cdot \sum_{t=0}^{k-1-j} \frac{1}{t !}\left[D^{(t)}\left\{\left(\frac{x-x_{i}}{\lambda(x)}\right)^{k}\right\}_{x=x_{i}}\right]\left(x-x_{i}\right)^{t}
$$

where the derivative operator indicates differentiation with respect to $x$. That $\hat{C}_{i j} \equiv C_{i j}$ is evident from the results of Lemma 1 . However, it is not obvious that this equivalence holds as $\hat{C}_{i j}$ contains a summation and is not nearly as compact in form as $C_{i j}$.

Schweizer [6] makes use of the results of Jacobi series to derive a formula for $p(x)$ in terms of the divided difference of $f$ of order $n j$, in which the arguments $x_{1}, \cdots, x_{i-2}, x_{i-1}, x_{i+1}, \cdots, x_{n}$ are repeated $j$ times and the argument $x_{i}$ is repeated $j+1$ times,

$$
p(x)=\sum_{j=0}^{k-1}\left\{\sum_{i=1}^{n} \frac{f\left[x_{1}^{j}, \cdots, x_{i-1}^{j}, x_{i}^{j+1}, \cdots, x_{n}^{j}\right]}{x-x_{i}} \frac{\pi(x)}{\pi^{\prime}\left(x_{i}\right)}\right\}[\pi(x)]^{j}
$$

This expression is symmetric in the $x_{i}$ and is identically equal to the expression given for $p(x)$ in (30). As it stands, Schweizer's formula is not in a particularly useful form since it does not involve an explicit representation of the interpolation polynomial. Algorithms for reducing Schweizer's formula to that of Spitzbart's may be found in the literature. See for example [4, p. 40] and [7, p. 44].

For a discussion of the remainder term associated with (30), the reader is referred to the work of Fort [3] in which interpolation is obtained in a form which involves an iterative process. 
8. Error Analysis. As stated previously, the right-hand expression of (18) is the error resulting from approximating the function $f(x, y)$ by the polynomial $q(x, y)$. This expression may be more compactly written as follows:

$$
\begin{aligned}
f(x, y)- & q(x, y) \equiv E(x, y) \\
& =\left(\frac{1}{2 \pi i}\right)^{2} \int_{C_{1}} \int_{C_{\Sigma}} \frac{[\lambda(s) \mu(y)]^{k}+[\lambda(x) \mu(t)]^{k}-[\lambda(x) \mu(y)]^{k}}{[\lambda(s) \mu(t)]^{k}(s-x)(t-y)} f(s, t) d s d t
\end{aligned}
$$

where the contours $C_{1}$ and $C_{2}$ are as previously defined.

No attempt will be made here to establish rigorous error bounds for the error expression (32). Perhaps one possible approach for establishing a bound on the error expression is to make use of a trivial extension of the important theorem from complex variables (cf., Churchill [2, p. 100]) which says that if $M$ is the upper bound of $g(z)$ on the curve $C$ and $L$ is the length of $C$, then

$$
\left|\int_{C} g(z) d z\right| \leqq M L \text {. }
$$

By experimenting with various contours $C$, satisfying the necessary criteria (see Section 3 ) imposed upon them, one should be able to establish a "least conservative" upper bound for (32).

In those cases when the integral changes sign frequently, as in many integral formulas for remainders, the method described above may tend to be very much of an over-estimation. In such cases the use of the dominant term in the Taylor expansion, or a possible application or extension of Montel's work in the complex plane for one variable to several real variables may be preferable.

The Boeing Company

Airplane Division

Renton, Washington

1. A. C. AhLin, "On error bounds for Gaussian cubature," SIAM Rev., v. 4, 1962, p. 25-39.

2. R. V. Churchild, Complex Variables and Applications, McGraw-Hill, New York, 1960, p. $158-159$.

3. T. Fort, Finite Differences and Difference Equations in the Real Domain, Clarendon Press, Oxford, 1948, p. 85-88.

4. F. B. Hildebrand, Introduction to Numerical Analysis, McGraw-Hill, New York, 1956.

5. H. E. SAlzer, \& G. M. Kimbro, Tables for Bivariate Osculatory Interpolation over a Cartesian Grid, Convair Astronautics, San Diego, 1958.

6. B. Schweizer, "A Symmetric generalization of the Lagrange interpolation formula." J. Math. Phys., v. 34, 1955, p. 157-159.

7. A. Spitzbart, "A generalization of Hermite's interpolation formula," Amer. Math. Monthly, v. 67, 1960, p. 42-46.

8. J. L. WALSH, "Interpolation and approximation by rational functions in the complex domain," Amer. Math. Society Colloquium Publications 20, 1935, p. 49-54. 